\title{
AN IMPROVED METHOD FOR CALCULATING CRITICAL SPEEDS AND ROTORDYNAMICS STABILITY OF TURBOMACHINERY
}

\author{
by \\ Brian T. Murphy \\ Research Assistant \\ and \\ John M. Vance \\ Dresser Industries Associate Professor \\ Department of Mechanical Engineering \\ Texas A\&M University \\ College Station, Texas
}

\begin{abstract}
Brian T. Murphy is currently a research assistant with the Turbomachinery Laboratories at Texas A\&M University. He received his B.S.M.E. degree in 1978 from the University of Florida. He is presently working toward his M.S., and eventually his Doctorate, both in Mechanical Engineering at Texas A\&M University. He has been conducting research for the past $2^{1 / 2}$ years in the area of rotordynamics, his primary field of interest. Projects that he is currently working on include development of an ultra-high energy density self-balancing flywheel and improvement of computer simulation methods for all types of rotating machinery.

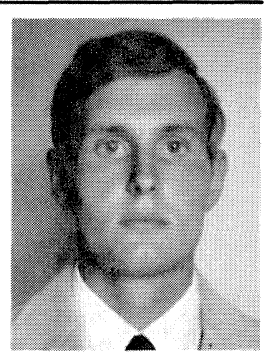

John M. Vance is a Professor of Mechanical Engineering at Texas AむM University. He received his B.S.M.E. (1960), M.S.M.E. (1964), and Ph.D. (1967) degrees from the University of Texas at Austin. He worked for five years as a mechanical engineer and engineering group leader in industry, including Armco Steel, Texaco Research and Tracor, Inc. He is active as a consultant to industry, and for five years was scien-

tific advisor to the U.S. Army on rotor dynamics and bearing design for turboshaft engines used in helicopters. He is a registered professional engineer in the States of Texas and Florida.

\section{ABSTRACT}

A number of the computer programs for rotordynamic stability and critical speed prediction in common use during recent years have been based on the works of Myklestad, Prohl and Lund. Programs of this type, called transfer matrix programs, employ complex variables when damping or crosscoupling is included in the model. Most use an iteration scheme which at times fails to converge with sufficient accuracy on some critical speeds, and has been known to completely miss critical speeds on occasion. It is shown in this paper that by rearranging the calculations performed in a transfer matrix program one can derive the characteristic polynomial for a complex rotor-bearing system, with no loss in generality. The modeling procedures are identical for the rotor and bearing/ foundations, including the effects of gyroscopics, damping, and any or all destabilizing influences which are linearized in the usual manner. With the characteristic polynomial known, critical speeds can be estimated and stability predicted with greater efficiency and with no fear of missing any modes. Such a program has been written, and a complete comparison between the two types of programs is shown.

\section{INTRODUCTION}

For years linear transfer matrix programs have been used by rotating machinery engineers for estimating critical speeds and predicting stability of complex rotor-bearing systems. The basic idea behind the workings of these programs was first put forth by Holzer [1] as a way of finding natural frequencies of torsional systems. This method was later adapted by Myklestad $[2,3]$ to calculate the natural frequencies of airplane wings coupled in bending and torsion. At about the same time Prohl [4] showed how this method could be applied to rotor-bearing systems complete with gyroscopic moments. The next significant advancement in the method was made by Lund $[5,6]$. Using complex variables, Lund showed how it could be applied to much more general rotor-bearing systems. By developing a more general formulation of bearing forces, Lund showed how system damping could be accounted for as well as self-exciting influences, such as oil whip and/or internal friction. It was with these latest developments that the method took on the full use of the general theory known as "The Linear Transfer Matrix Method."

Another item first introduced by Lund, and central to the subject of this paper, was a rather ingenious iteration scheme used to converge on the system eigenvalues. It is a NewtonRaphson approach and involves taking the derivatives of all equations used in the program. When programmed for a digital computer this technique works very nicely for many problems, but runs into trouble on others. One such pitfall is that it fails to converge with sufficient accuracy on some eigenvalues and has been known to completely miss one or more eigenvalues in some applications.

It is shown in this paper that by rearranging the calculations performed in a Lund-type program [6] one can calculate the coefficients of the characteristic polynomial for the rotorbearing system. The system natural frequencies, combined with their corresponding logarithmic decrements, are the roots of this polynomial. The logarithmic decrement provides the criteria for establishing system stability. With the polynomial known, these roots can be found and divided out in a more straightforward and efficient manner. Convergence can always be obtained and no critical speeds will be missed. 
The roots, or eigenvalues, are found in an iterative manner by both types of programs, but the polynomial program performs transfer calculations for the rotor-bearing system only one time in order to derive the characteristic polynomial. The iterations are then performed on the polynomial to find the roots. The number of iterations required to find a root is typically five to ten (rarely more than eleven) to achieve an accuracy of six digits. The Lund-type program also requires about the same number of iterations per root. However, all transfer calculations for the rotor-bearing system must be redone four times for each individual iteration. This difference accounts for a significant increase in computational efficiency for the polynomial program.

\section{PROCEDURE}

The rotor-bearing system is modeled in exactly the same manner as for the usual transfer matrix program [5]. The rotor is represented by concentrated masses connected by massless shafts (Figure 1). Bearings and foundations are modeled as forces acting oit the masses at the appropriate axial locations. The masses have the inertia properties of rigid circular cylinders and the shefts behave according to the Euler bending $\mathrm{a}_{\mathrm{d}}$ Timoshenko shear formulas. A shaft "station," see Figure 2, is normally considered to consist of a mass plus the shaft section immediately to its right.
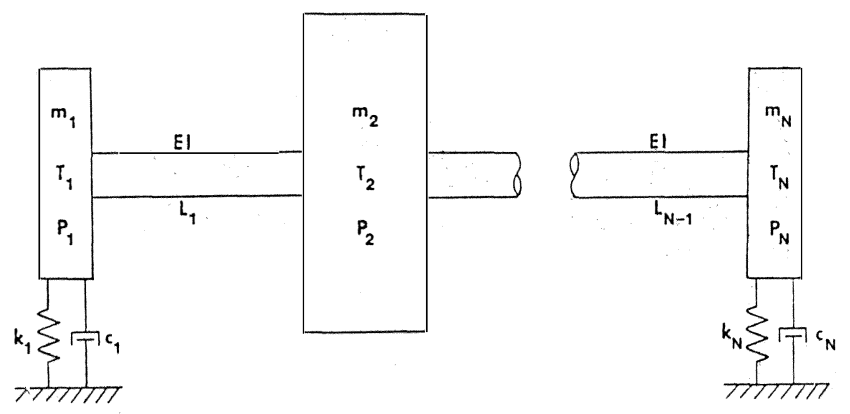

Figure 1. An N Inertia Rotor Bearing System.

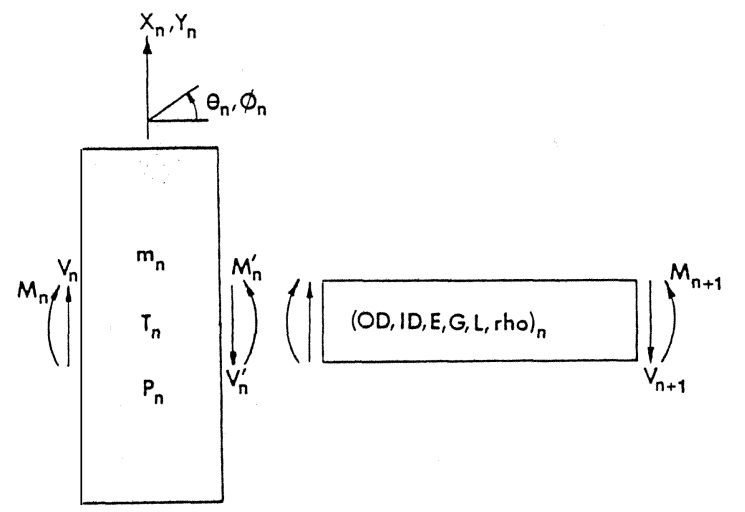

$$
\begin{gathered}
\text { Concentrated Mass Properties } \\
\text { m- amount of concentrated } \\
\text { mass } \\
T \text { - transverse (diametral) } \\
\text { moment of inertia } \\
\text { P - polar (axial) moment } \\
\text { of inertia }
\end{gathered}
$$$$
\begin{aligned}
& \text { Shaft Properties } \\
& \text { OD - outside diameter } \\
& \text { ID - inside diameter } \\
& \text { E - Young's Modulus } \\
& \text { G - Shear Modulus } \\
& \text { rho - mass density } \\
& \text { L - shaft length }
\end{aligned}
$$

Figure 2. A Single Shaft Station with Displacements $X$ and $Y$, Rotations $\theta$ and $\phi$, Shearing Force $V$, and Bending Moment $M$.
With the rotor-bearing system modeled as a connection of shaft stations, one assigns degrees of freedom (coordinates) at the junctions between the stations (i.e., at each concentrated mass). Linear differential equations are then written for each station and are arranged in matrix form. Since the equations are linear and homogeneous, the problem becomes an eigenvalue problem. Using the Laplace Transform, the matrices of differential equations are transformed into matrices of linear algebraic equations expressing the displacements and forces at the right end in terms of the displacements and forces at the left end [6]. The elements of these "transfer matrices" (or coefficients of the equations) are not constant but are actually polynomials in the system eigenvalue. (In the traditional transfer matrix program, the elements of the matrices are single numerical (complex) values, since a numerical estimate of the eigenvalue is made for the iteration calculations).

By definition, a transfer matrix "transfers" displacements and forces from one end of the station to the other. If the transfer matrices for two adjoining shaft stations are multiplied together using standard matrix multiplication, one obtains a single transfer matrix which fully represents the two stations. Since the elements of the two original matrices were polynomials, so will be the elements of the new matrix (and of correspondingly larger degree). Following this logic one can multiply together all the transfer matrices for the system and obtain a single transfer matrix which fully represents the entire system. The degree of the polynomial elements that make up this overall transfer matrix will be determined by the number of station $\mathrm{n}_{\mathrm{S}}$ in the original model.

Holzer [1] showed that the system eigenvalues can be found from the overall system transfer matrix by realizing that the forces at the shaft ends go to zero when the shaft vibrates at one of its natural frequencies. Thus, the determinant must vanish for the portion of the system transfer matrix that relates the forces at the right end to the displacements at the left end. Only at the natural frequencies can one have zero forces at the right end of the shaft even when the displacements are nonzero at the left end. Formulating the determinant of this submatrix yields the characteristic polynomial for the rotorbearing system.

To illustrate the procedure, consider the $\mathrm{N}$-inertia rotorbearing system shown in Figure 1, with symmetric bearing supports $\left(k_{n x}=k_{n y}, c_{n x}=c_{n y}\right)$. The transfer matrix for critical speeds across the $n$th mass $(\mathrm{n}=1,2, \ldots, N)$, from left to right, is

$$
\left\{\begin{array}{c}
\mathrm{X}_{\mathrm{n}}^{\prime} \\
\theta_{\mathrm{n}}^{\prime} \\
\mathrm{V}_{\mathrm{n}}^{\prime} \\
\mathrm{M}_{\mathrm{n}}^{\prime}
\end{array}\right\}=\left[\begin{array}{cccc}
1 & 0 & 0 & 0 \\
0 & 1 & 0 & 0 \\
-\mathrm{M}_{\mathrm{n}}(\mathrm{s}) & 0 & 1 & 0 \\
0 & -\mathrm{J}_{\mathrm{n}}(\mathrm{s}) & 0 & 1
\end{array}\right]\left(\begin{array}{c}
\mathrm{X}_{\mathrm{n}} \\
\theta_{\mathrm{n}} \\
\mathrm{V}_{\mathrm{n}} \\
\mathrm{M}_{\mathrm{n}}
\end{array}\right\},
$$

where

$$
\begin{gathered}
M_{n}(s)=m_{n} s^{2}+c_{n} s+k_{n} \\
J_{n}(s)=\left(T_{n}-P_{n}\right) s^{2} .
\end{gathered}
$$

The transfer matrix across the nth shaft section, from left to right, is

$$
\left\{\begin{array}{c}
\mathrm{X}_{\mathrm{n}+1} \\
\theta_{\mathrm{n}+1} \\
\mathrm{~V}_{\mathrm{n}+1} \\
\mathrm{M}_{\mathrm{n}+1}
\end{array}\right\}=\left[\begin{array}{cccc}
1 & l_{\mathrm{n}} & l_{\mathrm{n}}^{3} / 6 \mathrm{EI} & l_{\mathrm{n}}^{2} / 2 \mathrm{EI} \\
0 & 1 & l_{\mathrm{n}}^{2} / 2 \mathrm{EI} & l_{\mathrm{n}} / \mathrm{EI} \\
0 & 0 & 1 & 0 \\
0 & 0 & l_{\mathrm{n}} & 1
\end{array}\right] \quad\left(\begin{array}{c}
\mathrm{X}_{\mathrm{n}}^{\prime} \\
\theta_{\mathrm{n}}^{\prime} \\
\mathrm{V}_{\mathrm{n}}^{\prime} \\
\mathbf{M}_{\mathrm{n}}^{\prime}
\end{array}\right)
$$


Substitution of the left end boundary conditions, $\mathrm{V}_{1}=\mathrm{M}_{1}=0$, and successive multiplication of the transfer matrices from left to right will yield

$$
\left.\left\{\begin{array}{l}
\mathrm{V}_{\mathrm{n}}^{\prime} \\
\mathrm{M}_{\mathrm{n}}^{\prime}
\end{array}\right\}=\left[\begin{array}{ll}
\mathrm{d}_{11} & \mathrm{~d}_{12} \\
\mathrm{~d}_{21} & \mathrm{~d}_{22}
\end{array}\right] \quad \begin{array}{l}
\mathrm{X}_{1} \\
\theta_{1}
\end{array}\right\},
$$

where $d_{i j}$ is a polynomial in the eigenvalue s. In any contemporary computer program based on the Lund method, s will be assigned a numerical (complex) value for each iteration of the algorithm. Therefore, $\mathrm{d}_{\mathrm{ij}}$ will become a complex number, and the coefficients of the polynomial are lost.

In the polynomial program, the algorithm is written so as to preserve the coefficients of the plynomial, and the iteration is then performed using the polynomial equation

$$
\mathrm{D}(\mathrm{s})=\mathrm{d}_{11} \mathrm{~d}_{22}-\mathrm{d}_{12} \mathrm{~d}_{21}=0,
$$

the roots of which are the complex eigenvalues.

The degree of the characteristic polynomial depends on the complexity of the formulation (degrees of freedom per element) and the number of stations the shaft is divided into. The usual formulation has two displacements and two rotations per station, and thus the degree of the polynomial is eight per station (i.e., 72-degree for a nine station shaft model). It was originally thought that finding the roots of such large order polynomials might present serious numerical difficulties, in that the roots might be extremely sensitive to even the slightest computation errors in the coefficients. This was found not to be a problem and it can be shown analytically [7] that this situation is confined to only the higher modes. This seems reasonable since if one were to change, say, one of the masses by even a very small amount, this could cause a large change in the 100th critical speed but certainly not in the first or second critical speeds.

\section{RESULTS OF COMPARISON}

A complete comparison has been made of the polynomial type program with a Lund-type program written directly from reference [6]. The initial purpose of the comparison was to test the accuracy of the polynomial program against the Lund-type program. This was done using the Space Shuttle Hydrogen Turbopump as a model [8]. The model used (Figure 3) consists of nine stations and contains asymmetric bearing stiffnesses, destabilizing cross-coupling at three axial locations due to the pump interstage seals, and the aerodynamics of the turbines. With the cross-coupling coefficients used, the first forward mode will be unstable.

All roots were found to an accuracy of six digits in both programs and a direct comparison shows all corresponding roots to be identical within the limits of this accuracy. The comparison illustrates a considerable improvement by the polynomial program in efficiency of execution time. To find eight modes the Lund-type program required 27.6 seconds of execution time on a Prime 750 digital computer. Performing the same tasks and giving the same results, the polynomial program took just 5.4 seconds. Also, since the model has nine stations, it has 72 possible roots (36 conjugate pairs). The Lund-type program did not find all these roots since convergence breaks down for the higher modes. The polynomial program found all roots, requiring only one additional second of execution time. Thus, using the polynomial method one can find all the roots, be certain that none are missed, and for very little extra cost. It is felt that this is the greatest advantage of the polynomial program.
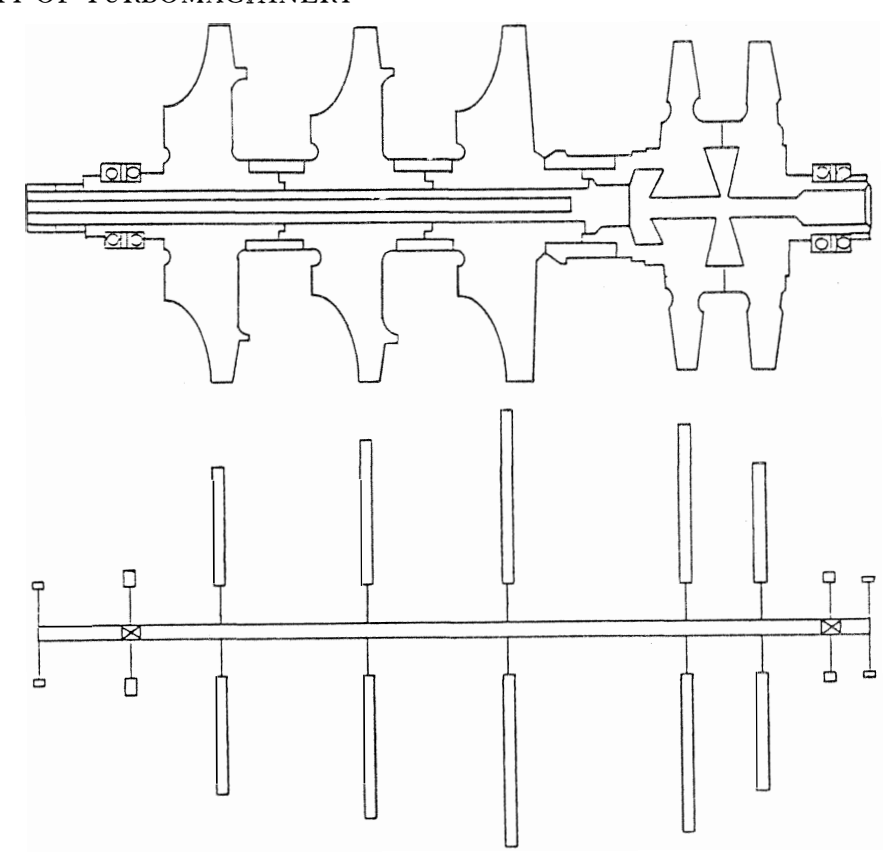

Figure 3. Space Shuttle Rotor and Computer Model.

The Space Shuttle model of Figure 3 can be considered to be of minimum complexity while still giving reasonably accurate results. The system is divided into just nine stations: one station for each disk (pump and turbine stages), one station for each bearing, and one for each portion of overhung shaft. This "minimum number of stations" modeling philosophy is used by some engineers because of its inherent simplicity. Another modeling philosophy that is widely used is to divide the rotor into many small stations in order to improve accuracy. To test the performance of the polynomial program on this type of model another comparison was made using an eight-stage centrifugal compressor typical of the petrochemical industry. This machine, weighing $1400 \mathrm{lbs}$, is supported on two tilt-pad bearings with a bearing span of 80.7 inches and is 103 inches in length overall. There also are a coupling and thrust collar overhung at the shaft ends. The rotor is modeled (Figure 4) as 35 stations supported in two flexible and damped bearings at stations 4 and 32 . Both the Lund-type program and polynomial program were executed to find the first eight modes. As for the Space Shuttle case, the results are identical within the precision obtained (six digits). The Lund-type program required 72.5 seconds to execute and the polynomial progiam required 34.3 seconds ( 26 seconds to derive the polynomial). Since the model has 35 stations there are 280 possible roots (140 conjugate pairs). The Lund-type program could not find all these roots, but the polynomial program did requiring an additional 30 seconds.

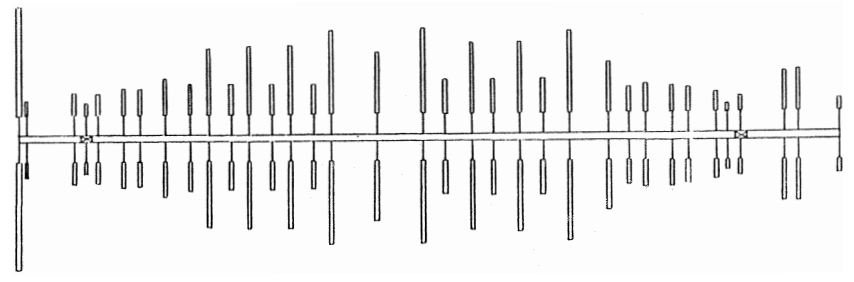

Figure 4. 35 Station Rotor Model for Eight Stage Centrifugal Compressor. 
As indicated above, the polynomial program executes faster than the Lund-type program. To investigate this further, a study was conducted using models of a uniform shaft supported in symmetric flexible bearings. Some of the results of this study are shown in Figures 5 and 6 . Figure 5 shows the ratio of execution times for finding roots for the two programs against the number of stations in the model and the number of roots found. It is seen that as more and more roots are sought the polynomial program gets better and better. This advantage is diminished by increasing the number of stations. Figure 5 also shows one of the problems sometimes encountered when running a Lund-type program. The erratic nature of two of the curves shown is caused by the inability of the Lund-type program to converge on the higher modes. Six-digit accuracy could not be obtained and so this requirement was cut back first to five diçits and then to four when necessary.

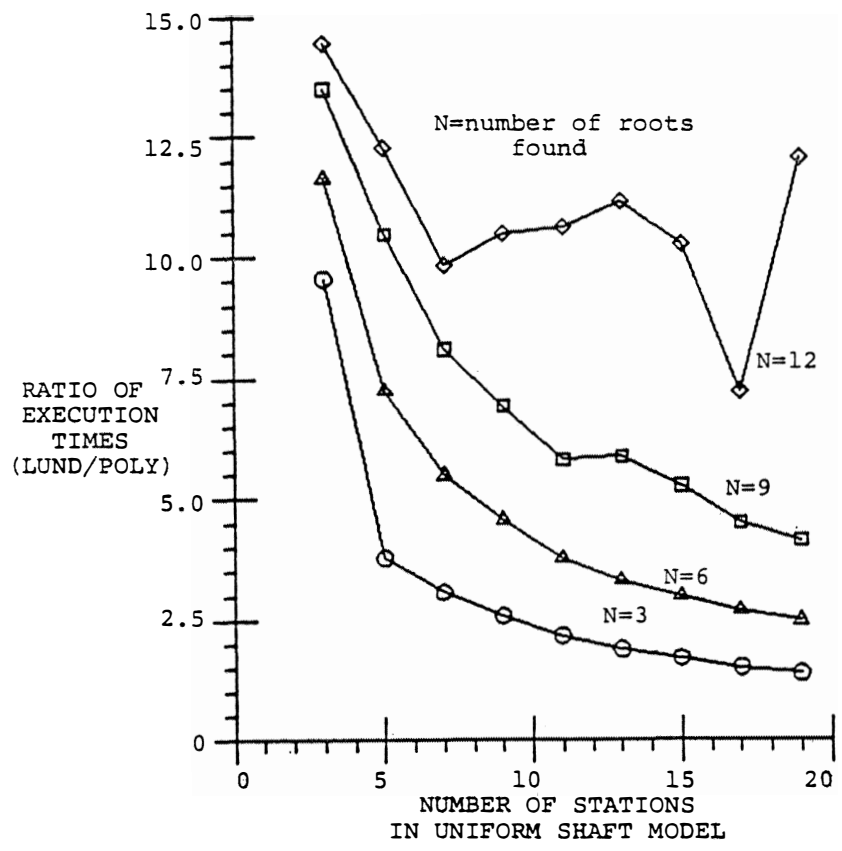

Figure 5. Comparison of Execution Time Required for Finding Roots.

Figure 6 shows why the advantage in execution time diminishes with the number of stations. The execution time of a Lund-type program increases rather linearly with the number of stations. Figure 6 shows that the time required to find the characteristic polynomial increases roughly with the square of the number of stations. For models with many stations this is most (more than half) of the total required execution time. The number of stations needed to make the two programs even is seen to be more than twenty (Figure 5) with the actual number depending on how many roots are sought.

As for mode shapes, the two programs calculate mode shapes in the same manner, with the polynomial program coming out ahead on execution time by about a factor of 2.75 (roughly constant). However, this result may be of small significance since the time required to find the mode shapes is only in the range of 10 percent of the total time required to run the program.

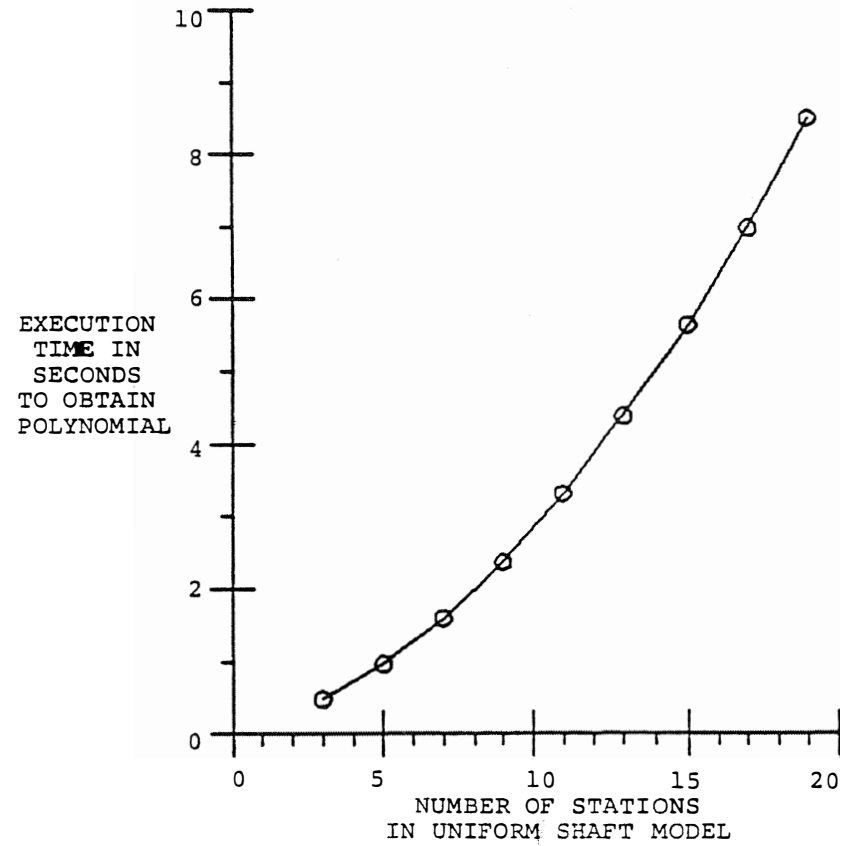

Figure 6. Time Required to Find Polynomial versus Number of Stations.

\section{USING THE PROGRAM}

Due to its faster execution time and reliable identification of all the eigenvalues, the polynomial program is especially well suited for the type of parameter studies that are often necessary for effective rotordynamic stability analysis. Many of the destabilizing forces which produce rotordynamic instability have not been quantitatively measured, and cannot be accurately predicted at present $[9,10,11,12]$. Therefore the most productive method of analysis for troubleshooting often is to vary the undetermined coefficients in repeated runs of the computer program until the field-observed critical speeds, the stability threshold speeds, and the instability frequencies are reasonably well simulated by the program. Subsequently, the program can be used to investigate the effect of changes in the design parameters which are known and which can be practically modified (e.g., bearing or seal stiffness and damping).

As an example, consider the eight-stage centrifugal compressor described earlier. This type of machine (multi-stage, high discharge pressure, high speed, with centrifugal impellers) has a history of costly rotordynamic instability problems, difficult to diagnose and to cure. There is a considerable body of experimental evidence $[9,10,11,12]$ which suggests that large destabilizing forces are produced by the dynamics of the working fluid around the impellers.

The forces on each impeller can be modelled in the program as linearized stiffness and damping, including crosscoupled terms, originally formulated by Lund [6]. Destabilizing effects from the working fluid often can be modelled using the cross-coupled stiffness coefficients $\mathrm{K}_{\mathrm{xy}}=-\mathrm{K}_{\mathrm{yx}}$. Figure 7 shows how the logarithmic decrement (stability indicator) for the $3090 \mathrm{cpm}$ mode of the compressor varies with the coefficient $K_{x y}$ over a range from $100 \mathrm{lb} /$ in to $10,000 \mathrm{lb} / \mathrm{in}$. The data for this curve was generated by the polynomial program in 514 seconds of computer time. The Lund program required 1090 seconds to generate the same data. 


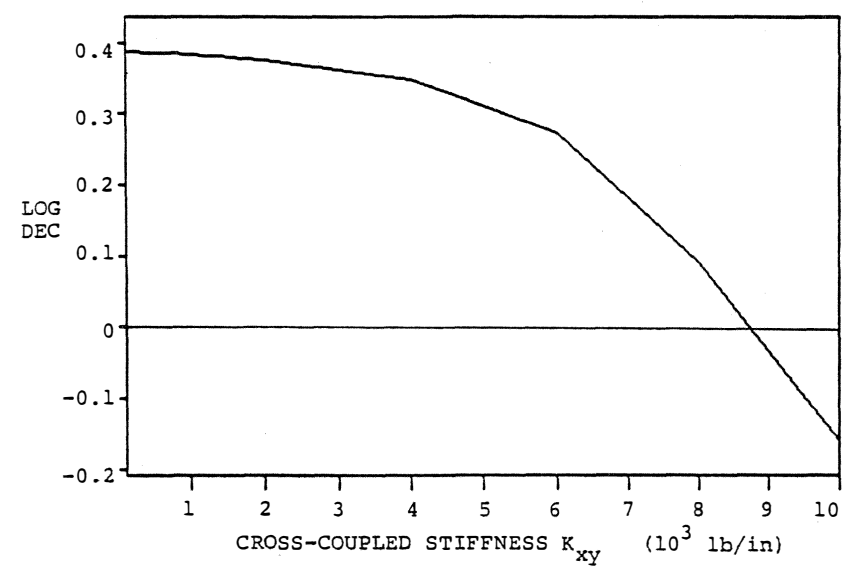

Figure 7. Stability Curve for First Forward Mode when Shaft Speed Equals Operating Speed.

Changing the design parameters in a compressor sometimes results in a shift of the instability from one mode to another [12]. In such a case, the greatest advantage of the polynomial program is that the "new" unstable modes and frequencies would not be missed by a failure to converge.

\section{CONCLUSIONS}

For stability and damped critical speed calculations on rotor-bearing systems, the polynomial program can perform the same tasks and give the same results as a Lund-type program while offering a significant savings in cost of execution. The polynomial program can also find all the systems' eigenvalues for only a very small additional cost (the Lund-type program is really not capable of finding all the eigenvalues at any cost). This capability eliminates the problem of sometimes missing eigenvalues and their associated modes. The advantage of faster execution increases as more roots are sought and diminishes as more stations are used.

The polynomial program is also much faster at finding mode shapes than the Lund-type program. However, this may be of little significance since the execution time required to find mode shapes is only a small part of the total time.

The polynomial program is especially well suited for the type of parameter studies that are often necessary for effective rotordynamic stability analyses, requiring repeated computer runs to determine the effective destabilizing forces acting in a machine.

\section{REFERENCES}

1. Holzer, Die Berechnung der Drehschwingungen (Berlin: Springer-Verlag, 1921).

2. Myklestad, N. O., “A New Method of Calculating Natural Modes of Uncoupled Bending Vibration of Airplane Wings and Other Types of Beams," Journal of Aeronautical Sciences, Vol. 11, No. 2 (April 1944), pp. 153-162.

3. Myklestad, N. O., "New Method of Calculating Natural Modes of Coupled Bending-Torsion Vibration of Beams," Trans. ASME, January 1945, pp. 61-67.

4. Prohl, M. A., “A General Method for Calculating Critical Speeds of Flexible Rotors," Journal of Applied Mechanics, Vol. 12, Trans. ASME, Vol. 67 (1945), pp. A-142-A-148.

5. Lund, J. W., and Orcutt, F. K., "Calculations and Experiments on the Unbalance Response of a Flexible Rotor," Journal of Engineering for Industry, Trans. ASME, Series B, Vol. 89, No. 4 (Nov. 1967), pp. 785-796.

6. Lund, J. W., "Stability and Damped Critical Speeds of a Flexible Rotor in Fluid-Film Bearings," Journal of Engineering for Industry, Trans. ASME, Series B, Vol. 96, No. 2 (May 1974), pp. 509-517.

7. Forsythe, G. E., Malcolm, M. A., and Moler, C. B., "Computer Methods for Mathematical Computations" (Englewood Cliffs: Prentice Hall, 1977), pp. 17-19.

8. Barret, L. E., Gunter, E. J., and Allaire, P. E., "The Stability of Rotor-Bearing Systems Using Linear Transfer Functions," School of Engineering and Applied Science, University of Virginia, Charlottesville, Virginia, Report No. UVA/464761/ME76/133, December 1976.

9. Wachel, J. C., "Nonsynchronous Instability of Centrifugal Compressors," ASME Paper 75-PET-22.

10. Vance, J. M., and Laudadio, F. J., "Nonsynchronous Instability of Centrifugal Compressors - Are All the Excitations Understood?", Journal of Engineering for Power, Trans. ASME, Vol. 103 (April 1981), pp. 288-293.

11. Fowlie, D. W., and Miles, D. D., "Vibration Problems with High Pressure Centrifugal Compressors," ASME Paper 75-PET-28.

12. Wachel, J. C., "Case Histories of Rotordynamic Instabilities in the Field," Short Course on Rotordynamics of Turbomachinery, Texas A\&M University, College Station, Texas, May 18-20, 1981. 
